Vassil Gjuzelev (Sofia)

\title{
LA QUATRIÈME CROISADE ET SES CONSÉQUENCES POUR LA BUlgarie MÉdiévale: LE TZAR KALOYAN, LES LATINS ET LES GRECS (1204-1207) ${ }^{1}$
}

La quatrième croisade est directement liée à l'histoire du Royaume bulgare; elle a un impact particulièrement intéressant et spécifique et des conséquences importantes pour lui. Dans une perspective plus proche, celles-ci peuvent être considérées à la lumière des événements survenus au cours de la période allant de 1204 à 1207 . Une telle analyse est nécessaire, parce que le dynamisme et la complexité des relations entre les puissances agissant à ce moment dans l'histoire du Sud-Est européen, ont conduit à plusieurs reprises les chercheurs aussi bien à une reconstruction incomplète et imprécise des événements en question, qu'à une interprétation incorrecte de leurs conséquences.

La guerre bulgaro-byzantine, déclanchée dès le début du règne du tzar Kaloyan (1197-1207), fut arrêtée en 1202 par la conclusion d'un traité de paix². En principe, il reconfirmait la légitimité du Royaume bulgare rétabli. La prise de Zara par les croisés en novembre 1202 et leur accord avec Alexis IV Ange, conclu dans le but de lui restituer le trône de son père, conduirent à une modification totale de la situation sur la péninisule balkanique. Leur avancée vers Constantinople et leur établissement devant ses enceintes (juin-juillet 1203) obligèrent le basileus Alexis III Ange (1195-1203) de rechercher l'aide militaire des Bulgares. Au début de juin 1203, afin de participer à des pourparlers, fut envoyé à Tărnovo le patriarche de Constantinople Jean Camatère (1198-1206) qui entra en contact avec le tzar bulgare et le chef de l'Eglise, l'archevêque Basile I ${ }^{\mathrm{er}}(1186-1232)^{3}$.

\footnotetext{
1 The paper is an abridged translation, supplemented with source quotations, of the previous author's text entitled Четвъртият кргстоносен поход, превземането на Цариград и БвлгароЛатинските отнотения 1204-1207 2., Pbg 28.2, 2004, p. 80-88.

2 Nicetas Choniates, Historia, rec. I.A. van Dieten, Berolini et Novi Eboraci 1975, p. 535 (cetera: Choniates, Historia); idem, Orationes et epistulae, rec. I.A. van Dieten, Berolini et Novi Eboraci 1972, p. 110; F. DöLGER, Regesten der Kaiserurkunden des oströmischen Reiches von 565-1453, vol. II, Regesten von 1025-1204, N 1661, p. 107.

3 Demetrius Chomatenus, Ponemata diaphora, rec. G. Prinzing, Berolini et Novi Eboraci 2002, p. 50-51, 426.
} 
Dans sa lettre au pape Innocent III (1196-1216), rédigée immédiatement après ces pourparlers, le tzar Kaloyan déclare avec fierté, que les Grecs avaient envoyé auprès de lui leur patriarche, qui au nom du basileus de Constantinople lui avait fait la proposition suivante:

Veni ad nos, coronabimus te in imperatorem et faciemus tibi patriarcham, quia imperium sine patriarcha non staret (Viens auprès de nous, nous te remettrons la couronne impériale et ferons pour toi patriarche, car un empire ne peut rester sans patriarche). ${ }^{4}$

Ayant compris la raison de la décision byzantine de faire des concessions quant à la reconnaissance de sa dignité de tzar et de celle de patriarche pour le chef de l'Eglise bulgare, le tzar Kaloyan refusa l'aide militaire demandée et se hâta de accélérer les pourparlers pour la conclusion d'une union avec l'Eglise de Rome. Il préféra assister en observateur aux événements qui avaient lieu sur les bords du Bosphore, contribuant indirectement à leur déroulement malheureux pour l'Empire byzantin.

Sous la pression des croisés, le 18 juillet 1203, Alexis III Ange quitta Constantinople et se retira dans la ville de Develte, située sur la côte ouest de la Mer noire et où antérieurement il avait préparé son refuge ${ }^{5}$. Dans sa tentative de préserver ses droits de souverain, il n'avait pas choisi par hasard cette ville qui se trouvait à la frontière avec la Bulgarie. A propos de ces événements, il serait intéressant d'attirer l'attention sur un récit, figurant dans L'histoire des basileus georgienne (XIII ${ }^{\mathrm{e}}$ s.), lequel, en mélangeant la vérité à la légende, explique la catastrophe byzantine:

Lorsque les Francs apprirent que les Grecs avaient reçu une aide de l'Est, vinrent les Vénitiens et conquirent la capitale, tandis que le malheureux Alexis s'enfuit en Bulgarie auprès de son gendre. Quand le tzar bulgare, son gendre, le vit, il l'emmena dans une de ses forteresses, jeta devant lui une grande quantité d'or et lui dit: Voici Alexis, c'est ce que tu voulais. Prends cet or au lieu de nourriture et d'eau, car tu as perdu la maison royale chrétienne et as détruit l'autocratie grecque. Dans ces conditions, le malheureux Alexis mourut de faim, privé de l'aide de Dieu. ${ }^{6}$

Il est évident qu'au cours de l'été 1203, Alexis III Ange pour la deuxième fois demanda de l'aide militaire aux Bulgares, mais celle-ci lui fut refusée. C'est pour cette raison que très rapidement il quitta Develte pour s'installer à Andrianople, mais sa tentative d'en faire le centre d'un mouvement de restauration de son pouvoir à Constantinople fut très vite déjouée. Le 1 août 1203, Alexis IV (1203-1204)

4 Преписката на папа Инокентия III с българите, еd. И. Дуйчев, ГСУ.ИФФ 37, 1942, p. 31 (cetera: Преписката на папа Инокентия III с българите).

5 Choniates, Historia, p. 549, 556; Georgius Acropolites, Historia, rec. A. Heisenberg, Lipsiae 1903, p. 6 (cetera: Acropolites).

6 С.Г. КАухчишвили, Грузинские источники по истории Византии, vol. I, Тбилиси 1974, p. $146,148$. 
fut proclamé et couronné basileus byzantin. La légitimité de ce couronnement ne fut pas reconnue par le tzar bulgare Kaloyan? ${ }^{7}$. Il suivait attentivement les événements qui se déroulaient dans l'Empire byzantin en ruines, en essayant de la comprendre au mieux et de prendre la position qui correspondait à ses intérêts.

Ayant hâté, à la fin de 1203, le déroulement des pourparlers pour la conclusion d'une union avec l'Eglise de Rome, Kaloyan décida de miser sur une alliance avec les croisés qui se trouvaient devant les enceintes de Constantinople. Cela correspondait à sa politique essentiellement anti-byzantine, qu'il menait avec une grande conséquence. Selon le témoignage de Robert de Clari, en février 1204, par une ambassade auprès des chevaliers latins, le souverain bulgare leur proposa de mettre à leur disposition une armée de cent mille personnes afin qu'ils puissent prendre Constantinople. Le refus du Conseil des barons latins à cette proposition, exclua la participation bulgare à la future opération ${ }^{8}$. Cette démarche de Kaloyan était tout à fait dans la ligne de sa politique. Il essayait non seulement de tâter le terrain quant à ses rapports avec le nouveau facteur des événements dans les Balkans, mais visait une participation active et équitable au partage de l'héritage byzantine. De toute évidence, il ne se rendait pas compte que celle-ci était partagée d'avance entre les croisés et les Vénitiens, lesquels, pour cette raison, n’avaient aucun besoin d'un autre allié.

La prise de Constantinople par les Latins (le 12 avril 1204), l'élection de Baudouin de Flandre comme empereur du nouvel empire et la mise en pratique du Partitio terrarum imperii Romanie deja ébauchée, modifièrent radicalement la situation. Assuré par le pape Innocent III dans sa lettre du 25 février 1204 d'être couronné très bientôt par un légat pontifical qui lui remettrait le sceptre royal (sceptrum regale) et le diadème royal (regiam diadema), le tzar Kaloyan essaya de nouveau d'arranger d'une manière pacifique ses rapports avec les nouveaux maîtres de Constantinople. Nicétas Choniatès (1155-1216) note que lorsqu'il envoya une ambassade pour montrer son amitié, on lui répondit de parler dans les missives qui leur ferait parvenir non pas comme un basileus à ses sujets, mais comme un serviteur à ses maîtres. Sinon ils leveraient une armée et n'auraient pas de difficulté à mettre à sac la Mésie (La Bulgarie) qu'il gouvernait non pas de droit, mais en tant qu'apostat qui s'etait séparé des romaioi ${ }^{9}$. D’une façon plus détaillée ce fait est raconté dans Les actes du pape Innocent où l'on raconte que lorsque le tzar Kaloyan

avait reçu la nouvelle de la prise de Constantinople, il envoya une ambassade auprès des Latins et une lettre leur proposant de faire la paix, mais ils lui répondirent avec dédain qu'il n’y aurait jamais de paix avec lui s'il ne rendait les terres qui appartenaient à l'Empire de Constantinople et qu'il avait

7 Geoffroy de Villehardouin, La conquete de Constantinople, 202, ed. et trans. E. Faral, Paris 1938 (cetera: Villehardouin).

8 Robert de Clari, La conquete de Constantinople, 64, ed. Ph. Lauer, Paris 1924 (cetera: Clari).

9 Choniates, Historia, p. 612. 
conquises par la force. Il leur répondit qu'il avait sur ces terres davantage de droits qu'eux-mêmes en avaient sur Constantinople. Car lui-même s'était rendu les terres que ses ancêtrs avaient perdues, tandis qu'ils avait conquis Constantinople, qui n’avait jamais été leur possession. ${ }^{10}$

C'etait donc comme si, avec la prise de Constantinople, les Latins avaient hérité de la doctrine byzantine qui traitait le Royaume bulgare comme un royaume surgi à la suite d'une sécession $\left(\dot{\alpha} \pi \circ \sigma \tau \alpha \sigma^{\prime} \alpha\right)$ du territoire grec. Cela allait à l'encontre de la conception politique bulgare d'une continuité et de rétablissement d'une tradtion étatique qui avait déjà existé. S'étant incarné en héritiers de l'empire byzantin et de sa doctrine politique, les Latins ne pouvaient pas compter sur une tolérance et une soumission de la part de Bulgares.

Au cours de l'été 1204, le tzar Kaloyan fit sa troisième et dernière tentative de se réconcilier avec les Latins, juste au moment où ils entreprirent le partage réel des possessons balkaniques. Cette tentative eut lieu lors de la rencontre du souverain bulgare avec l'un des chefs de la quatrième croisade, le noble français Pierre de Bracheux, décrite en détails par Robert de Clari. Le dialogue entre les deux fait comprendre que les Latins avaient une justification idéologique et une explicaton historique de l'agressivité qu'ils montraient: cette terre, jadis, aurait appartenu à leurs ancêtres de Troie la Grande et ils y seraient venus pour la reconquériri ${ }^{11}$. Les événements qui s'ensuivirent montrèrent que les Bulgares connaissait bien la Légende de Troie mais non dans sa version contemporaine mise à jour par les Latins.

Entretemps, le légat pontifical, le cardinal Léon Brancaléon, enfin arrivé à Tărnovo, le 7 novembre 1204 sacra l'archaviêque de Tărnovo Basile $\mathrm{I}^{\mathrm{er}}$ qui devint primas des Bulgares et des Valaques (primas Bulgarorum et Blachorum) et le jour suivant il couronna Kaloyan roi de toute la Bulgarie et de la Valachie (rex totius Bulgarie et Blachie $)^{12}$. Dans sa lettre au pape Innocent III, envoyée peu de temps après, le souverain bulgare qui pensait non sans fondements avoir reçu le titre de tzar (imperator), formula d'une manière franche et précise sa position ferme aussi bien à l'égard du Royaume hongrois qu'à l'égard du nouvel Empire latin en le prévenant sans aucune ambiguïté:

De même, quant aux Latins qui s'emparèrent de Constantinople, j'écris à votre Sainteté de leur faire savoir de se tenir loin de mon royaume et de le respecter et alors mon royaume ne leur fera aucun mal. Mais s'ils entreprennent quelque chose contre mon royaume et qu'ils ne le respectent pas et tuent de ces gens qui lui sont soumis, que Votre Sainteté ne soupçonne pas mon royaume et que tout sois libre de tout [soupçon]. ${ }^{13}$

L'adhésion du Royaume bulgare à l'union avec l'Eglise de Rome et la recherche du rôle médiateur du pape pour l'arrangement des relations avec

${ }^{10}$ Innocentii III papae gesta, [in:] PL, vol. CCXIV, col. 147-148.

11 ClaRi, 106.

12 Преписката на папа Инокентия III с бблгарите, р. 65-67.

13 Преписката на папа Инокентия ІІІ с българите, р. 66. 
l'Empire latin restèrent sans résultat. Il était de plus en plus difficile de maîtriser l'agressivité croissante des Latins. Selon la version aragonaise de la Chronique de Morée ( $\mathrm{XIV}^{\mathrm{e}}$ s.) le roi bulgare craignait que l'empereur Baudouin, qui se trouvait à la frontière de ses terres, pût (y) entrer et conquérir son royaume comme il l'avait déjà fait avec celui des Grecs $^{14}$. Selon Geoffroy de Villhardouin, lors de la campagne en Thrace orientale pendant l'été de 1204, le marquis Boniface de Montferrat avait adressé à Baudouin les paroles suivantes: Dirigeons-nous, si tel est votre désir, contre Johanes qui est roi de la Bulgarie et de la Valachie et possède d'une manière illégitime une grande partie de la terre ${ }^{15}$. Le parti-pris anti-bulgare ferme des chefs de la quatrième croisade se voit dans le partage des terres de Romania qui comprenait des régions et des cités déjà inclues dans les frontières du Royaume bulgare: une grande partie de la Thrace et du littoral de la Mer noire, la région des Rhodopes et la Macédoine avec des villes et des places fortes comme Philippople, Sténimachos, Constantia, Béroé, Mesembria, Anchialo, Prilep, Castoria, Măglen, Ohrid etc. ${ }^{16}$ Dans les réponses aux ambassades et aux propositions bulgares transparaissait de plus en plus nettement l'agressivité de la politique latine envers le Royaume bulgare.

Au demeurant, à la fin de 1204 et au début de 1207, le conflit entre la Bulgarie et l'Empire latin paraissait inévitable. Selon le témoignage de Geoffroy de Villhardouin, à la veille de la future guerre, des envoyés des Grecs de toutes les villes du pays proposaient leur aide au tzar Kaloyan en lui faisant savoir qu ils le feraient empereur (il le feroient empereor), que tous se soumettraient à lui comme à leur maître, tandis qu'il les gouvernerait comme ses sujets ${ }^{17}$. Quelle que soit l'interprétation de ce témoignage par la recherche scientifique actuelle, il est évident que dans la situation aux Balkans, qui fut extrêmement défavorable pour les romaioï, ces derniers, pour la première et la dernière fois, furent obligés de reconnaitre le tzar bulgare comme leurs souverain et prêtèrent serment de le servir.

Dans la bataille d'Adrianople qui eut lieu le 14 avril 1205, un an après la prise de Constantinopple, les Bulgares, alliés aux Coumans et aux Grecs, firent subir une défaite écrasante à l'armée latine, firent prisonnier l'empereur Baudouin et l'amenèrent dans leur capitale Tărnovo ${ }^{18}$. Le coup porté à l'Empire latin paraissait mortel: l'empereur fut décapité, la fleur de sa chevalerie périt. Toute la terre de Thrace se trouvait entre les mains des Bulgares, la cavalerie de leurs alliés, les

\footnotetext{
${ }^{14}$ Libro de los fechos et conquistas del principado de la Morea, publiée et traduite pour la première fois par A. Morel-Fatio, Genève 1885, p. 16 (cetera: Libro de los fechos et conquistas del principado de la Morea).

15 Villehardouin, 276.

${ }_{16}$ Венециански документи за историята на България и българите от XII-XV в., еd. В. ГЮзЕЛЕВ, София 2001, p. 14-15.

17 Villehardouin, 333.

18 В.Н. ЗЛАТАРски, История на българската държава през средните векове, vol. III, Второ българско иарство. България при Асеневци (1187-1280), София 1940, р. 224-227; И. БожиЛов, В. ГюзЕЛЕВ, История на средновековна България VII-XIV век, София 1999, p. 453-454.
} 
Coumans, atteignit Constantinople (seules les villes de Rodosto et de Selivrie demeuraient latines), les marchands, les chevaliers et les sergents s'embarquèrent sur un navire vénitien pour quitter Constantinople, le vieux doge vénitien Enrico Dandolo fut tué ${ }^{19}$. Le destin de l'Empire latin, qui inopinément s'était enclavé dans l'histoire du Sud-Est européen, semblaient entièrement entre les mains du tzar bulgare Kaloyan.

La bataille d'Andrianople de 1205, l'un des événements de l'histoire européenne les plus largement rapportés et commentés par l'historiographie occidentale $\mathrm{du} \mathrm{XIII}^{\mathrm{e}}-\mathrm{XV}^{\mathrm{e}} \mathrm{s}$., a eu une importance extraordinaire et des conséquences sans précédent pour le Royaume bulgare. Tout au début de son rétablissement, il eut subitement la possibilité d'écraser l'empire latin et de prendre possession de l'héritage byzantin dans les Balkans. Au bout de trois siècles, renaissait la chimère du tzar Siméon le Grand (893-927): la création d'un royaume bulgaro-grec avec Constantinople pour capitale. Trois facteurs s'opposèrent à la réalisation de ce rêve: 1) la puissance réduite, mais pas anéantie, des chevaliers latins; 2) le soutien qu'ils recevaient de l'Eglise de Rome et de l'Occident catholique et 3) le mouvement anti-bulgare des Grecs de la Péninsule balkanique. Dans cette situation complexe et pleine de surprises, le tzar Kaloyan, qui jusqu'alors avait agi en diplomate habile et en excellent chef miltaire, fit preuve d'une incohérence dans ses actions et de confusion. Avec son armée, composée de Grecs et de Bulgares il se dirigea soudain contre le marquis de Thessalonique, Boniface de Monferrat ${ }^{20}$. Cette démarche était une réponse à la conquête provisiore de la ville, en juin 1205, par le souverain de Prosec, Tzuzmène, qui avait profité de l'absence du marquis ${ }^{21}$. Alors, le tzar bulgare, qui avait pris successivement Seres, Ber (Véréa) et les autres villes soumises au marquis, au lieu de marcher sur Thessalonique, se dirigea vers Plovdiv où il imposa son pouvoir, soutenu par les Bulgares - popelicans (pauliciens) et combattant la résistance des romaioï, menés par Alexis Aspiète. Tout de suite après, le tzar se rendit à Tărnovo où il écrasa le complot des traitres qu'il punit de peines lourdes et de nouveaux procédés de mise à mort. C'est à ce moment qu'il décida de changer d'attitude envers ses alliés et de faire aux romaioi une guerre meurtrière et implacable à cause de leur pérfidie, traitrise et inconséquence ${ }^{22}$. La résistance grecque à Plovdiv et le complot de Tărnovo furent les premières fissures sérieuses dans la brève alliance bulgaro-greque. Le serment des Grecs de Thrace de reconnaitre le tzar bulgare comme leur basileus se perdit dans le passé...

Etant informé de la grave défaite des Latins à Andrinople, le pape Innocent III, par la voie diplomatique, essaya de sauver leur empire - en fait son propre enfant - qui brusquement s'était trouvé au seuil de la perdition. Dans sa lettre de juin

19 Villehardouin, 352-388.

20 Villehardouin, 389, 392-394; Choniates, Historia, p. 618-622.

${ }^{21}$ Choniates, Historia, p. 619; Libro de los fechos et conquistas del principado de la Morea, p. 17.

22 Choniates, Historia, p. 625-628; Villehardouin, 399-401. 
1205, adressée au tzar Kaloyan, il lui demande de libérer Baudouin et de conclure une paix réelle et solide avec les Latins. Il alla jusqu'à le menacer d'une avancée des Hongrois et d'une nombreuse armée de croisés qui bientôt serait partie des pays occidentaux pour la Grèce ${ }^{23}$. Il est peu probable que les menaces du pape aient troublé le tzar bulgare, ce qui l'aurait mené à faire la paix avec les Grecs.

La campagne militaire bulgare du début de 1206 se caractérise par le pillage des villes et des forteresse en Thrace orientale et la déportaton de sa population grecque en Bulgarie. L'aristocratie grecque, renonçant à son alliance avec Kaloyan sous prétexte qu'il ne respecte aucun accord, conclut une convention avec les Latins $^{24}$. Son chef, Théodore Vranas, et son allié, Henri d'Hainaut, qui le 20 août 1206 (après la nouvelle de la mort de Baudouin à Tărnovo) fut proclamé empereur, pour la première fois déployèrent une activité anti-bulgare ${ }^{25}$.

Dans cette situation d'isolement, le tzar Kaloyan fut obligé au début de 1207 de conclure une alliance avec les Grecs de l'Asie mineure unis sous le sceptre du basileus de Nicée Théodore I ${ }^{\text {er }}$ Lascaris (1204-1222). L'alliance entre le Royaume bulgare et l'Empire de Nicée eut une importance capitale pour la liquidation ultérieure de l'Empire latin ${ }^{26}$.

Pour arrêter la menace bulgare, lors de leur rencontre à Kypsela en août 1207, l'empereur Henri et le marquis Boniface de Monferrat se mirent d'accord sur l'organisation d'une campagne commune contre la Bulgarie ${ }^{27}$. Cependant, le 4 septembre 1207, près de Mosynoupolis, le marquis Boniface de Monferrat fut attaqué et tué par les Bulgares de la région. Sa tête coupée fut envoyée au tzar Kaloyan pour qui c'était une des plus grandes joies qu'il avait éprouvées ${ }^{28}$. De cette manière, les Bulgares causèrent la mort des deux représentants les plus éminents de la quatrième croisade. Par ironie de l'histoire, une mois après la mort de Boniface de Monferrat, le tzar Kaloyan fut assasiné par des conjurés devant les murs de Thessalonique. La tradition ecclésiastique attribua sa mort à saint Démètre de Thessalonique ${ }^{29}$.

En fait, la quatrième croisade, surtout à ses débuts, eut des conséquences très spécifiques et pas entièrement négatives pour le Royaume bulgare. La prise de Constantinople et la mise en place initiale de l'Empire latin se déroulèrent au cours d'une période pendant laquelle ce royaume était en pleine expansion; il cherchait à s'affirmer aussi bien sur le plan balkanique qu'européen et était gouverné par

\footnotetext{
${ }_{23}$ Преписката на папа Инокентия III с българите, р. 69-70.

${ }^{24}$ Choniates, Historia, p. 628; Villehardouin, 422-423.

25 Villehardouin, 441.

26 Villehardouin, 459; V. GJuzelev, Bulgarien und Kaiserreich von Nikaia (1204-1261), JÖB 26, 1977, p. $145-146$.

27 Villehardouin, 495-497.

${ }^{28}$ Villehardouin, 498-499; A. Ceruti, Un codice del monastero cistercense di Lucedio, ASI 8, 1881, p. 378, an. 4: Obiit Bonifacius marchio, fundator huius domus.

${ }_{29}$ В.Н. ЗлАТАРСКИ, op. cit., p. 252-259, 581-587.
} 
l'un des souverain médiévaux les plus remarquables - le tzar Kaloyan. Exclu d'une manière arrogante par les barons latins du partage du territoire byzantin, celui-ci devint l'ennemi acharné de la présence occidentale dans l'espace balkanique et lui porta des coups militaires et politiques irréparables. Il obligea le nouvel empire, dès sa fondation, à prendre une position défensive et contribua largement à la conservation et à la consolidation des restes de l'étatisme byzantin, en particulier de celui de l'Empire de Nicée et du Despotat d'Epire ${ }^{30}$. Le résultat historique objectif de ses actions contraste avec le jugement grec de cette époque et les appréciations ultérieures. Il reste dans la tradition historique byzantine avec les incursions dévastatrices de ses troupes en Thrace et en Macédoine et avec la déportation d'une population grecque dans les frontières de la Bulgarie. Selon Georges Acropolite (1217-1282) et Théodore Scoutariote (deuxième moitié du XIII ${ }^{\mathrm{e}} \mathrm{s}$.):

Kaloyan se vengeait, comme d'ailleurs il le disait lui-même, des souffrances que l'empereur avait infligées aux Bulgares. Et en déclarant que Basile s'appelait Bulgaroctone, il se nommait lui-

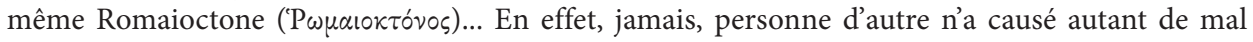
à la terre grecque, de sorte qu'on lui donnât le surnom de chien: tous l'appelaient Johanes le Chien $\left(\sum \kappa \nu \lambda o i ̈ \omega \alpha \dot{\alpha} \nu \eta \varsigma\right) .{ }^{31}$

Il serait intéressant de noter que dans la mémoire littéraire bulgare, ses victoires contre les Latins occupent une place secondaire. Le patriarche Euthyme (1375-1394) le présente comme le conquérant du Royaume grecque (Гръчьское царство) еxténué et comme l'envahisseur d'une grande partie de la terre grecque et de ses villes ${ }^{32}$. De même, dans le Synodicon de l'Église bulgare ( $\mathrm{XIII}^{\mathrm{e}}-\mathrm{XIV}^{\mathrm{e}} \mathrm{s}$.) on glorifie sa mémoire pour les nombreuses victoires contre les Grecs et les Latins (на Грькы и Фругы $)^{33}$.

Malgré l'union avec l'Eglise de Rome de 1204, de fait et objectivement, le Royaume bulgare agissait contre l'Empire latin de Constantinople, prenant par là une position de defenseur de l'orthodoxie. Dans la tradition historique bulgare et slave $\left(\mathrm{XIII}^{\mathrm{e}}-\mathrm{XIV}^{\mathrm{e}} \mathrm{s}\right.$. $)$ on porta un jugement fort catégorique sur ce fait:

En l'an 6712 [=1204] Constantinople fut pris par les Latins, parce que l'empereur Théodore Lascaris, soit n'y était pas, soit ne pouvait pas leur tenir tête. Mais en ces temps-là, l'Église orthodoxe avait son défenseur - le tzar bulgare... Le Royame grecque se divisa en deux: toute la Théssalie, l'Illyrie et Thessalonique se trouvaient sous la domination de Théodore Lascaris, tandis qu' à Ponte, Galathie, Bythinie et en Asie régnait Kaloyan Vatatzes, celui qui craignait Dieu. ${ }^{34}$

${ }_{30}$ G. Ostrogorsky, Geschichte des byzantinischen Staates, München 1963, p. 353-354.

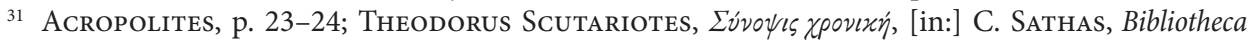
graeca medii aevi, vol. VII, Parisiis 1894, p. 459-460.

32 Werke des Patriarchen von Bulgarien Enthymius (1375-1393), nach den besten Handschriften hrsg. von E. KaluŽniaCKi, Wien 1901, p. 56, 95, 197.

33 И. Божилов, А. ТотомановА, И. Билярски, Борилов Синодик. Издание и превод, София 2010 [= ИК], p. 150, 20 - 151, 2.

34 А. Попов, Обзор хронограбов русской редакиии, vol. I, Москва 1866, p. 190-192; vol. III, Москва 1869 , p. 33-34, 36-37, 144-145. 


\section{Abstract. The Fourth Crusade and Its Consequences for the Medieval Bulgaria: Kaloyan, the Lat- ins and the Greeks (1204-1207)}

The analysis of the Bulgarian-Latin relations between 1204 and 1207, i.e. in the second half of Tsar Kaloyan's reign, leads to conclusions as follows: 1 . the Bulgarian monarch played a major role in forcing the newly-created Latin empire to adopt a defensive policy in the political struggles in the Balkan Peninsula; 2. thus, the Tsar contributed to retaining and, in consequence, consolidating the remnants of the Byzantine empire (Nicaea, Epirus-Thessalonica); 3. in the Byzantine memory the actions of Kaloyan were evaluated negatively, as times of plundering the Byzantine territories and relocations of its population into Bulgaria; 4. for the later Bulgarian historiography the Tsar's wars with the Latins were of secondary significance, and despite the official union with the Roman church he was remembered as a defender of the Orthodox faith.

Vassil Gjuzelev

Bulgarian Academy of Sciences

1 „15th of November” Str.

1040 Sofia, Bulgaria

byzcongress2011@yahoo.com 